\title{
A CAR T-cell recipe for success
}

Promising outcomes have been obtained in trials of CD19-targeted chimeric antigen receptor (CAR) T cells for the treatment of patients with B-cell malignancies, although responses were variable owing to the heterogeneous composition, differentiation status and transgene expression levels of the T cells infused. To overcome these limitations, Michael Jensen and collaborators established anti-CD19 CAR T-cell infusions with a defined composition and tested them in a clinical trial, results of which have been published in Blood.

They used a mixture containing $\mathrm{CD} 4^{+} / \mathrm{CD} 8^{+}$ CAR T cells (in a 1:1 ratio) with uniformly high levels of anti-CD19 CAR expression (achieved through lentiviral transduction) and limited in vitro terminal differentiation (owing to treatment with homeostatic cytokines). "This effort", Jensen explains, "resulted in a very consistent product profile across patients, with a $100 \%$ manufacturing reproducibility."

A total of 45 patients with relapsed or refractory CD19-positive acute lymphoblastic leukaemia (ALL) - with a median age of 12.3 years (1.3-25.4 years) - were enrolled in the phase I study to test these CAR T cells. A minimal residual disease-negative complete response was observed in $93 \%$ of patients who received CAR T cells (40/43), and in $100 \%$ of those who received a fluradabine/ cyclophosphamide lymphodepleting regimen (14/14). Jensen considers these response rates "a new milestone in the field". All remisions occurred by day 21 . Of the 40 patients achieving a complete response, 18 experienced disease relapse, of which seven were associated with a loss of CD19 expression.

At 12 months, the event-free survival and overall survival were $50.8 \%$ and $69.5 \%$, respectively. The overall incidence rates of cytokine-release syndrome (CRS), severe CRS, neurotoxicity, and severe neurotoxicity were $93 \%, 23 \%, 49 \%$, and $21 \%$, respectively; these toxicities were manageable, and no treatment-related deaths were reported. This CAR T-cell product is being tested in a phase II trial in both children and young adults; if confirmed, the remission rates observed so far will be good news for the oncology community.

Diana Romero

ORIGINAL ARTICLE Gardner, R. A. et al. Intent to treat leukemia remission by CD19CAR T cells of defined formulation and dose in children and young adults. Blood http://dx.doi.org/ 10.1182/blood-2017-02-769208 (2017) 